\title{
Formation de la couche limite autour d'un pipeline soumis à l'action de la houle
}

\author{
Mouazé dominique (doctorant), Pr. Bélorgey michel \\ Groupe Mécanique des Fluides et Génie Côtier - Centre de Géomorphologie \\ 24, rue des Tilleuls - 14000 CAEN-France
}

\section{Résumé}

L'étude de l'écoulement autour d'un cylindre, placé horizontalement près du fond d'un canal à houle, présente des intérêts importants: Tout d'abord pour la connaissance des phénomènes complexes engendrés et ensuite pour la modélisation hydrodynamique autour de telles structures. Les investigations expérimentales consistent en l'étude des couches limites générée par la houle sur le fond et autour du cylindre. L'étude du champ des vitesses s'effectue au moyen d'un vélocimètre Doppler Laser. La sonde de rétro-diffusion est localisée à l'intérieur du pipeline de telle sorte que les faisceaux soient focalisés dans l'écoulement. Cette fonction permet l'acquisition des vitesses prés de la surface du cylindre avec une grande résolution spatiale. Enfin, l'approche d'une modélisation des données expérimentales permet une meilleure compréhension des évolutions spatiales et temporelles des différentes couches limites étudiées.

\begin{abstract}
Detecting wave-induced flow velocities around a smooth circular cylinder, fixed horizontally near the bed of a middle-scale wave channel, represents an important part in physical modeling and in the knowledge of the complex hydrodynamic phenomena under such structures. The experimental investigation consists of the measurement of the velocity field, with Laser Doppler Anemometer system, especially on the boundary layer under the waves and around the cylinder. The Laser head is located inside the structure and the beams are focussed on the flow to permit the velocity measurement near the cylinder surface with a high spatial resolution.
\end{abstract}

\section{Introduction}

La proximité du fond ainsi que les effets de séparation, conduits par la couche limite, jouent un rôle significatif sur les efforts d'un pipeline à l'échelle naturelle. De plus, la houle ajoute une combinaison complexe de phénomènes tels que l'accélération du fluide, le mouvement orbital, ainsi que l'épaisseur de la couche limite des vagues. Afin d'étudier l'effet de proximité d'une paroi, différents rapports d'espacement ont été choisis. Les investigations, concernant la formation du sillage, s'étendent sur plusieurs régimes fixés par le nombre de Keulegan-Carpenter. La première partie des résultats s'étend sur les profils de vitesse des vagues ainsi que ceux de la couche limite près du fond. L'analyse des vitesses autour du cylindre donne une quantification des caractéristiques spatiales et temporelles de la couche limite parallèlement à l'évolution de la surface libre. 


\section{Système expérimental}

Les essais sont réalisés dans un canal à houle dont les dimensions sont en longueur 120 mètres, en largeur 2.2 mètres et en hauteur 2.5 mètres. Une pente en enrochement, située à l'extrémité du canal permet de réduire la réflexion des vagues. Un batteur plan à piston permet de générer une houle de type régulière. Le cylindre consiste en un tube creux en aluminium de la largeur du canal et dont le diamètre est $210 \mathrm{~mm}$. Comptetenu du mouvement de translation et de rotation du volume de mesure, un système de coordonnées cylindriques est adopté. Les faisceaux Laser, conduits par fibres optiques placées à l'intérieur de la structure, doivent

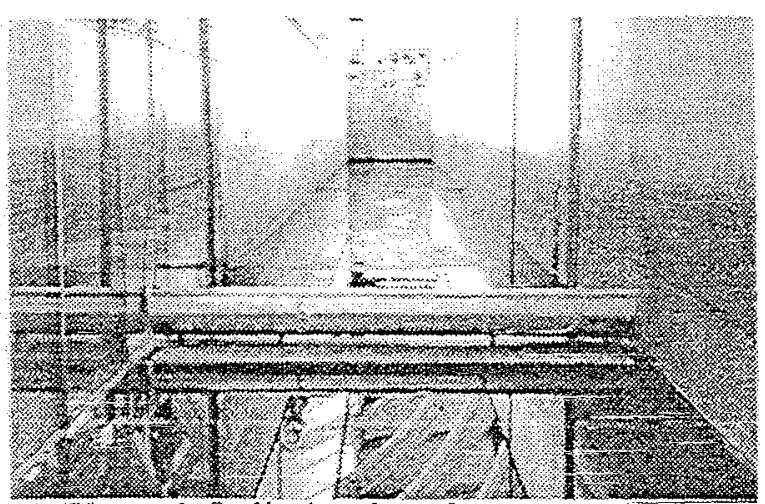

Photo.1 Cylindre dans le canal à houle

Photo. 1: Cylinder in the wave flume passer à travers une fenêtre transparente encastrée sur la surface afin d'atteindre le point de mesure hors du cylindre. Ce dernier est rempli d'eau afin de limiter le nombre de milieux d'indice de réfraction différents. Cependant, cette technique a nécessité l'emploi d'un caisson étanche renfermant le moteur pas à pas pour le déplacement de la tête optique. L'ensemble du dispositif est décrit par les auteurs Dursthoff et al. (1997). La résolution spatiale est de $0.11 \mathrm{~mm}$ en tenant compte de l'inclinaison du volume de mesure. La partie centrale du cylindre est mise en rotation avec un pas de $20^{\circ}$. De plus, 19 points sont acquis le long de chaque rayon à partir de la surface de la fenêtre et jusqu'à $25 \mathrm{~mm}$ de celle-ci.
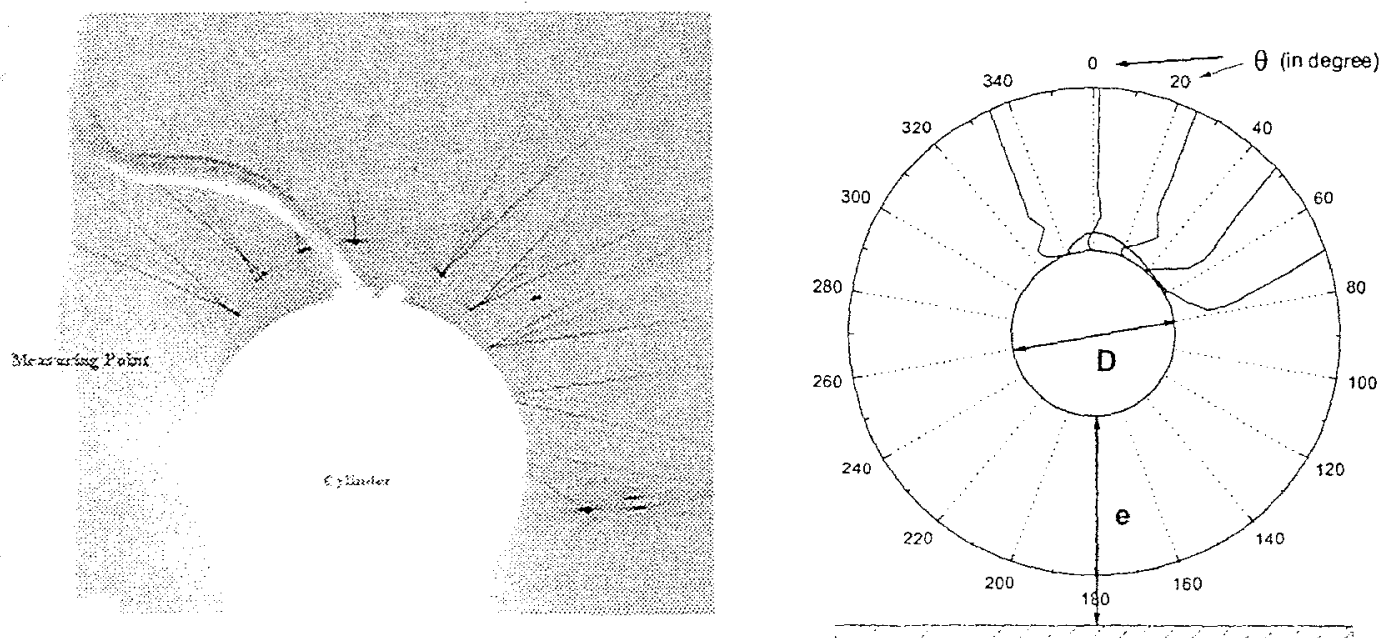

Fig.1 Différentes positions d'inclinaison de l'axe de mesure Fig. I Tilted positions $\left(\theta=0^{\circ}, 20^{\circ}, \ldots, 340^{\circ}\right)$ of the Laser beam axis

\section{Conditions expérimentales}

Pour une période fixe, l'amplitude de la houle est ajustée afin d'obtenir le nombre de Keulegan-Carpenter voulu. Toutes les caractéristiques des vagues sont déterminées 
suivant la théorie de Stokes au deuxième ordre. Le tableau suivant (Tab.1) présente les paramètres principaux des conditions expérimentales:

\begin{tabular}{|c|c|c|c|c|}
\cline { 2 - 5 } \multicolumn{1}{c|}{} & $\mathrm{W}_{1}$ & $\mathrm{~W}_{2}$ & $\mathrm{~W}_{3}$ & $\mathrm{~W}_{4}$ \\
\hline $\mathrm{KC}$ & $\mathbf{1 . 5}$ & $\mathbf{2 . 8}$ & $\mathbf{4 . 8}$ & $\mathbf{8 . 8}$ \\
\hline $\mathrm{T}(\mathrm{s})$ & $\mathbf{2 . 0}$ & 3.0 & 3.5 & 5.0 \\
\hline $\mathrm{H} / \mathrm{d}$ & $\mathbf{0 . 1 5}$ & 0.15 & 0.22 & 0.18 \\
\hline $\mathrm{L}(\mathrm{m})$ & $\mathbf{7 . 5}$ & 8.7 & 12.1 & 15.2 \\
\hline $\mathrm{U}_{\mathrm{cmax}}(\mathrm{m} / \mathrm{s})$ & $\mathbf{0 . 1 7}$ & 0.21 & 0.29 & 0.37 \\
\hline Regimes of flow & $\begin{array}{c}\text { Separation/ } \\
\text { turbulent } \\
\text { boundary layer }\end{array}$ & $\begin{array}{c}\text { symetric pair of } \\
\text { vortices }\end{array}$ & $\begin{array}{c}\text { asymetric pair of } \\
\text { vortices }\end{array}$ & $\begin{array}{c}\text { vortex shedding } \\
\text { (for e/D>0.1) }\end{array}$ \\
\hline
\end{tabular}

Tab. 1 Caractéristiques des vagues

Tab.l Characteristics of the waves

La hauteur d'eau s'élève à un mètre. Les régimes d'un écoulement oscillant autour d'un cylindre sont établis par Sumer et al. (1997). La vitesse $U_{c m a x}$ représente la vitesse maximale au cours d'une période à la position fictive du centre du cylindre, i.e. à $20 \mathrm{~cm}$ du fond du canal. Elle permet de déterminer le nombre de Keulegan-Carpenter, nombre sans dimension, caractéristique du type de l'écoulement. La structure est placée à trois hauteurs différentes afin d'étudier l'influence du fond et en particulier la suppression de l'allée tourbillonnaire. Ces élévations correspondent aux rapports d'espacement e/D suivants: $\mathrm{e} / \mathrm{D}=0.03,0.08$ et 0.5 .

\section{Acquisition et analyse des données}

Nous limiterons la présentation de l'étude aux résultats de la vague $\mathrm{W}_{1}$.

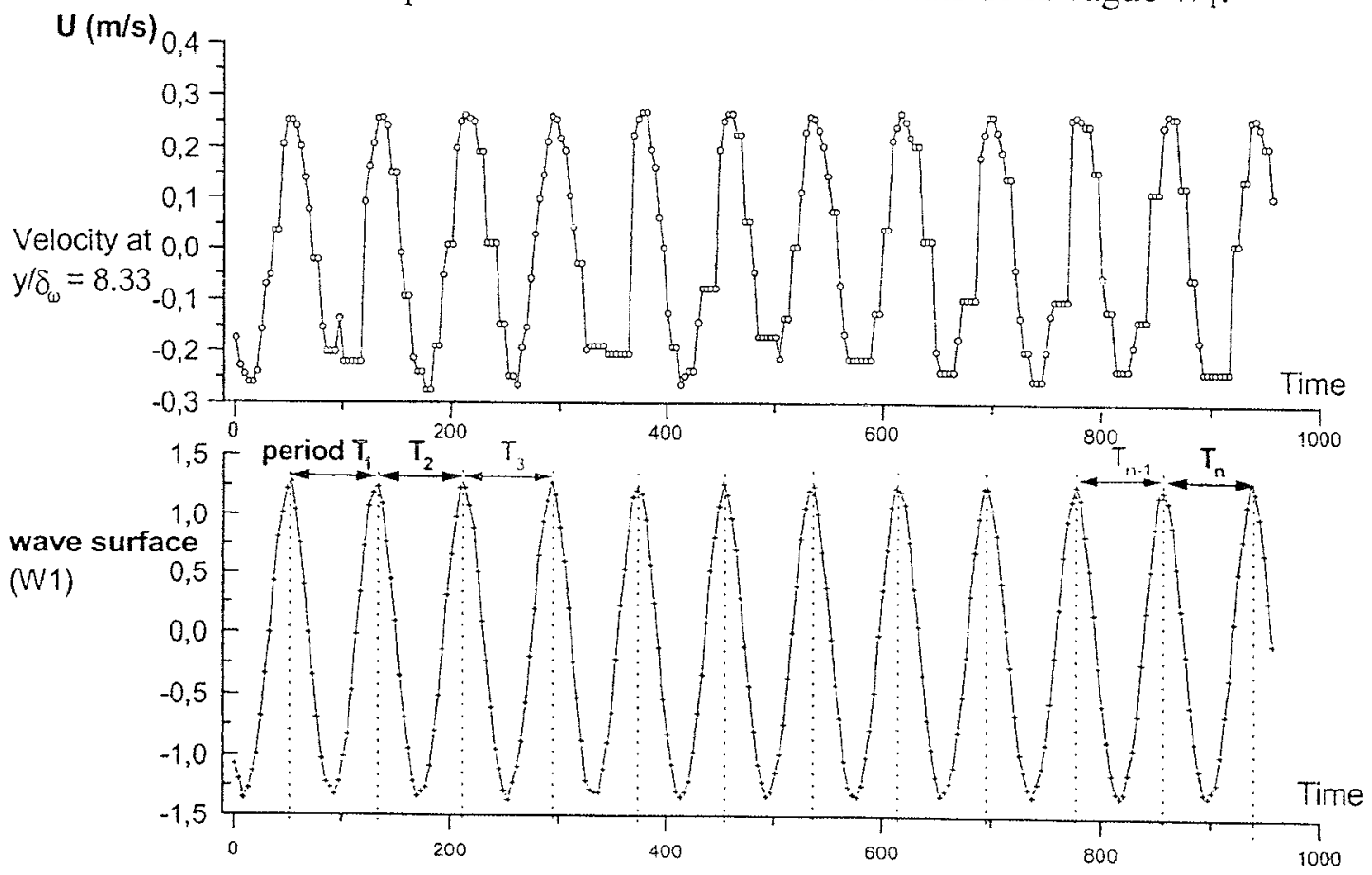

Fig.2 Signaux de vitesse et de surface libre

Fig. 2 Velocity and wave surface signals

Les propriétés statistiques des vitesses et de la surface libre sont calculées à l'aide d'une méthode de phases moyennes. La figure précédente (Fig.2) représente un enregistrement de la vitesse à $25 \mathrm{~mm}$ de la surface du cylindre et, parallèlement, l'état de la surface libre 
correspondante. L'enregistrement de surface libre est décomposé en $N$ périodes. Celles ci sont alors superposées afin d'obtenir un profil moyen de houle et des vitesses pour l'ensemble des phases. La figure 3 montre les opérations successives. Ces courbes sont lissées avec un filtre FFT d'ordre 8 afin d'éliminer les points incohérents sans signification physique. Une propriété de l'électronique d'instrumentation est de conserver en mémoire la valeur d'une vitesse jusqu'à la prochaine validation. De cet artéfact résulte l'apparition de "marches" dans le profil des vitesses lorsque le taux d'acquisition est trop faible. L'analyse des données inclut le rejet de ces paliers lors du calcul des valeurs moyennes.
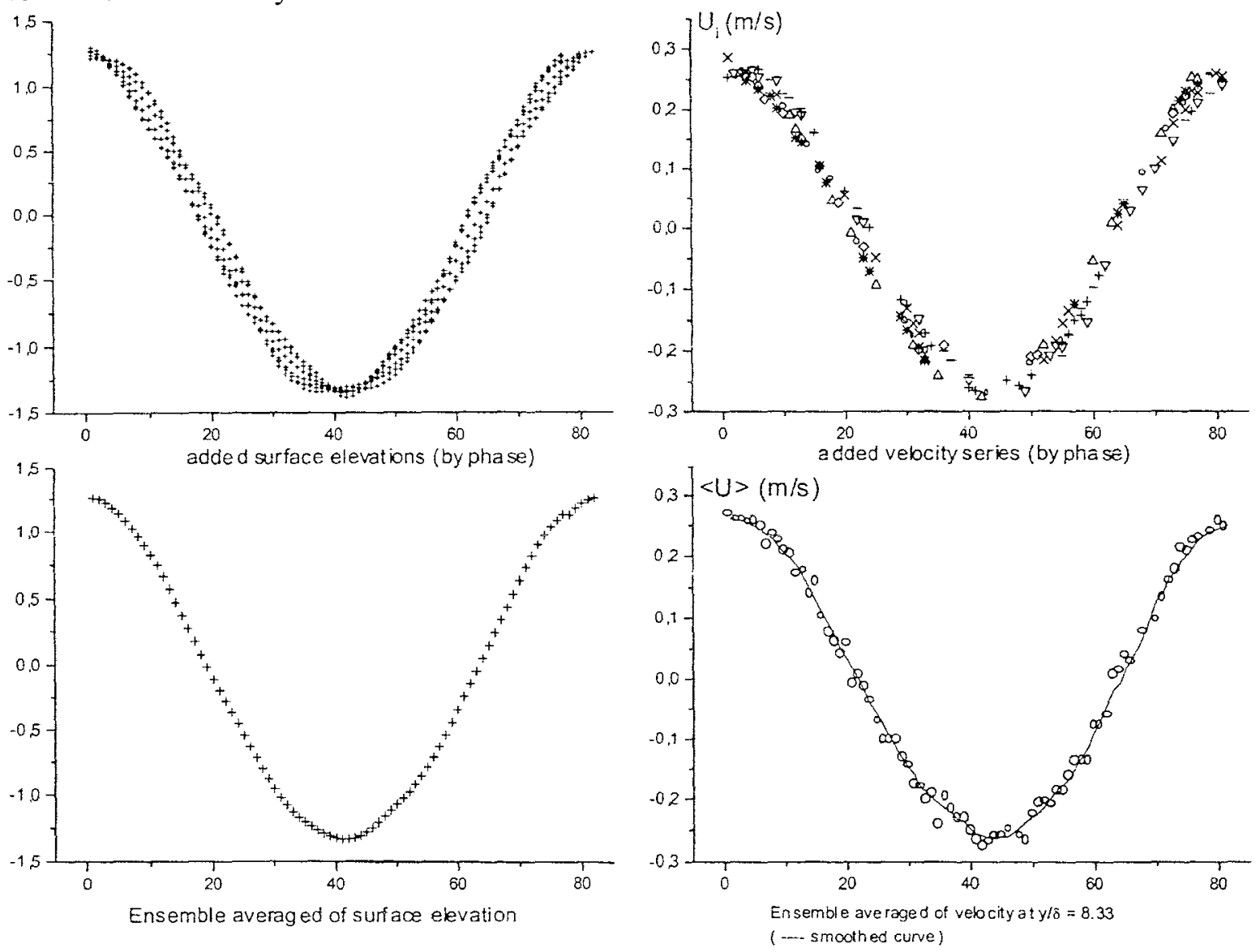

Fig.3 Méthode d'analyse par phase moyenne

Fig. 3 Phase ensemble averaging method of data analysis

L'enregistrement temporel est relatif à l'acquisition par une jauge de surface libre placée dans l'axe de l'optique d'émission Laser (pour les vagues) ou bien dans l'axe du cylindre (pour la couche limite). Le pas de déplacement prés du fond du canal est ajusté afin d'étudier également la couche limite générée par la houle seule. La précision de la résolution spatiale $(0.11 \mathrm{~mm})$ permet l'accès à de nombreux points dans l'épaisseur de couche limite et ainsi de déterminer la rugosité du fond. La réflexion intense par la paroi au point de croisement des faisceaux produit une saturation de l'électronique de réception. Avec cette considération pratique et à l'aide d'une référence optique précise, l'élévation du fond $z_{0}$ est évaluée à $0.8 \mathrm{~mm}$. Ainsi $z_{0}=\mathrm{k} / 30=0.8 \mathrm{~mm}$, avec $\mathrm{k}$ la rugosité selon Nikuradse. La figure suivante montre l'évolution des vagues moyennes pour différentes hauteurs à partir du fond: 


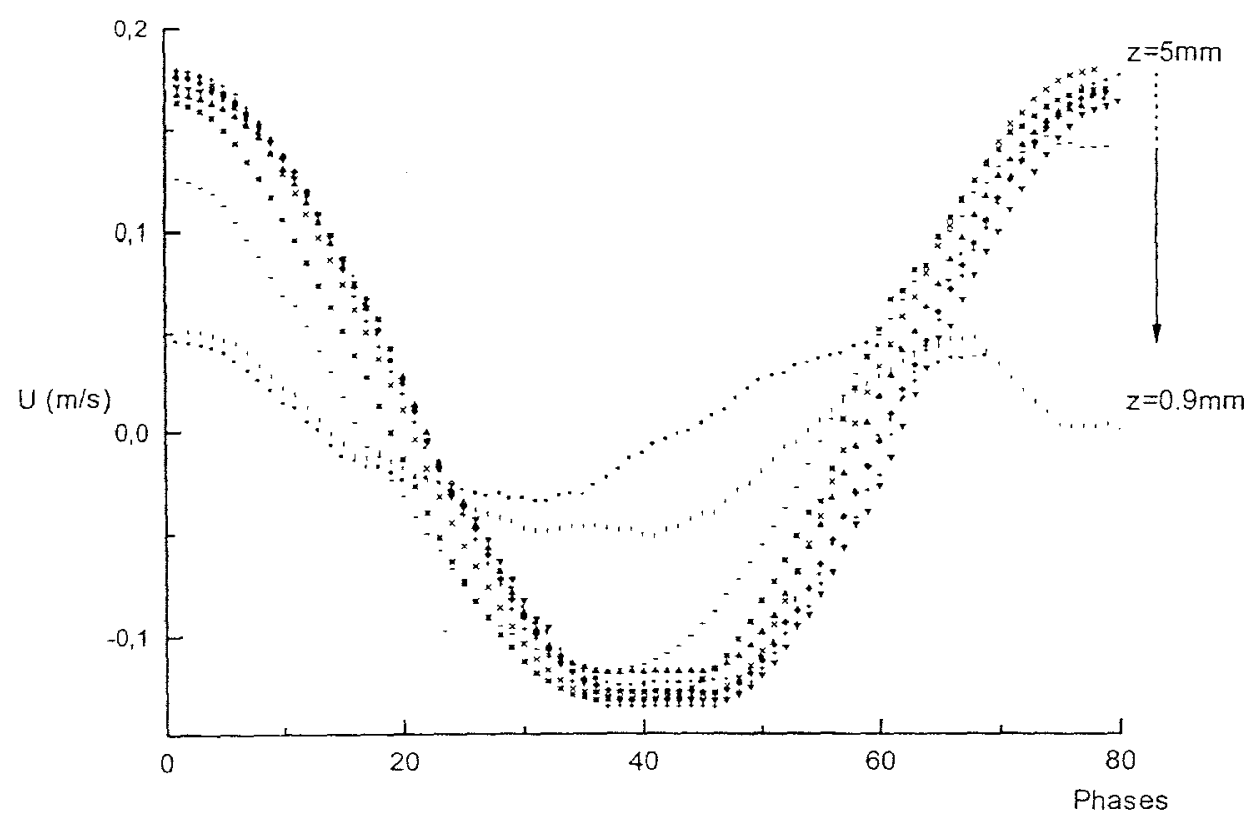

Fig.4 Superposition de profils de vitesse par phase (vague $\mathrm{W}_{1}$ )

Fig 4 Superimposed velocity profiles by phase (wave $W_{l}$ )

A partir de la figure 4, on peut déduire le déphasage entre les profils théoriques et expérimentaux des vitesses dans la houle sur l'ensemble de la hauteur d'eau.

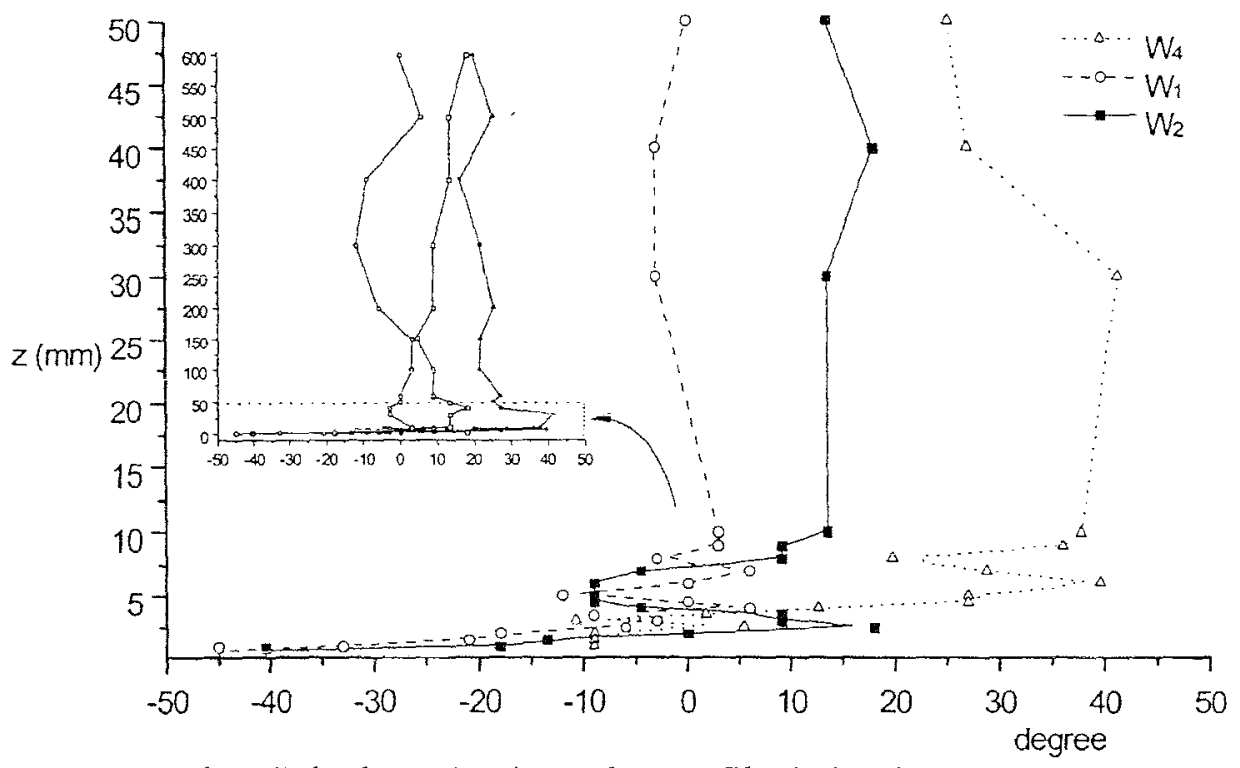

Fig.5 Décalage de phase des profils de houle (vague $W_{1}$ )

Fig.5 Phase shift along the water depth

Le détail des résultats donnés sur la figure 5 met en exergue trois zones principales: L'avance de phase dans la zone proche de la surface libre montre l'asymétrie des vagues $\mathrm{W}_{2}$ et $\mathrm{W}_{4}$. A $50 \mathrm{~mm}$ du fond du canal, ce décalage est encore plus marqué. Cette tendance s'inverse complètement prés de la paroi où les profils de vitesses accusent un retard de phase conséquent. Ce comportement sera confirmé particulièrement dans la couche limite ce qui incitera à augmenter les perturbations (oscillations) des profils de vitesses prés du fond en créant des couches de cisaillement. Ce point sera abordé plus loin. 


\subsection{Résultats des profils globaux et de la couche limite des vagues}

Afin de comparer plus précisément les valeurs expérimentales et celles issues d'une théorie de la houle, la vitesse horizontale des particules fluides est calculée à partir d'une approximation au second ordre développée par Stokes. Comme le montre le graphe suivant (Fig.6), les phases extrêmes de la houle (crête et creux) sont en bonne concordance avec la théorie utilisée. Cependant, les profiles expérimentaux sont sousestimés particulièrement pour les phases intermédiaires (phases 20 et 60 sur le diagramme) et présentent une surélévation des vitesses (overflow) prés du fond. Ces différences peuvent être expliquées par la valeur élevée du gradient de vitesse et par le développement d'un courant résiduel lors de ces phases. La réflexion des vagues par l'amortisseur de houle, qui est estimée à $6.5 \%$ pour le cas de la vague $W_{1}$, doit jouer également un rôle important.

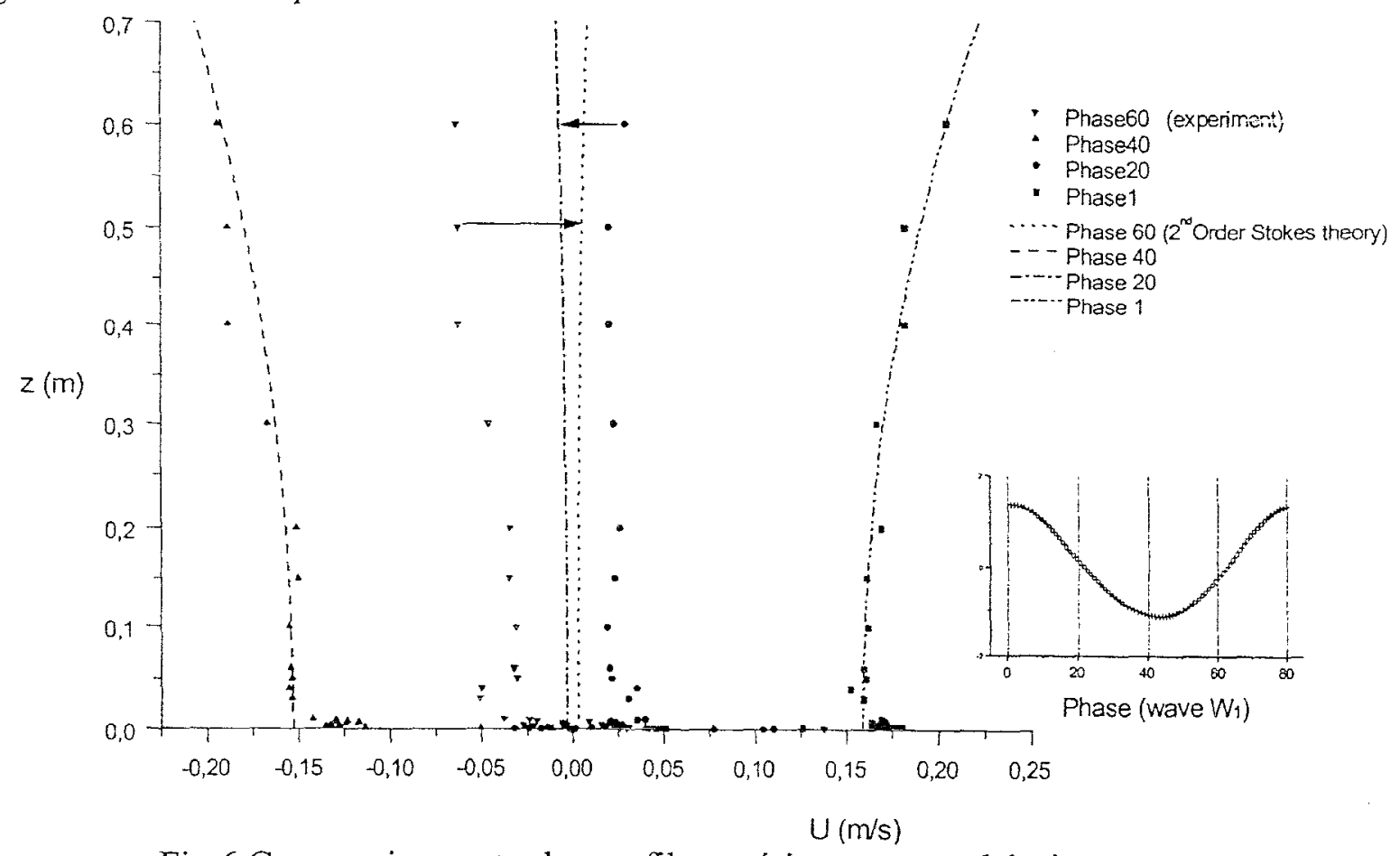

Fig.6 Comparaison entre les profils expérimentaux et théoriques

Fig. 6 Comparison between experimental velocity profile and the Stokes theory

Des données importantes peuvent être tirées de ces profils globaux. Tout d'abord, la vitesses maximale de l'écoulement libre $U_{o m}\left(U_{o m}=0.20 \mathrm{~m} / \mathrm{s}\right)$ qui est utilisée pour les critères de similitude. Enfin, la vitesse maximale $\mathrm{U}_{\mathrm{cm}}$ à la position fictive du centre du cylindre détermine le nombre sans dimension de Keulegan-Carpenter: $\mathbf{K C}=\frac{\mathbf{U}_{c m} \mathbf{T}}{\mathbf{D}}$, avec D le diamètre du cylindre.

\subsection{Couche limite générée par la houle}

On admet que les effets visqueux soient confinés à une fine couche le long du fond du canal. De nombreuses définitions de cette couche limite ont été postulées pour le cas d'un écoulement plan oscillant. Très peu de modèles prennent place en écoulement générée par la houle particulièrement par manque de données expérimentales. Jonsson (1980) définit l'épaisseur de couche limite $\delta$ comme la plus 
petite longueur à laquelle la vitesse égale celle de l'écoulement libre pour l'extension maximale du mouvement oscillant. Selon Sumer (1991), l'épaisseur de couche limite est la hauteur minimale à laquelle le gradient des vitesses s'annule. Un profil expérimental typique de la couche limite de houle est montré sur la figure 7. Les différentes définitions y sont également représentées:
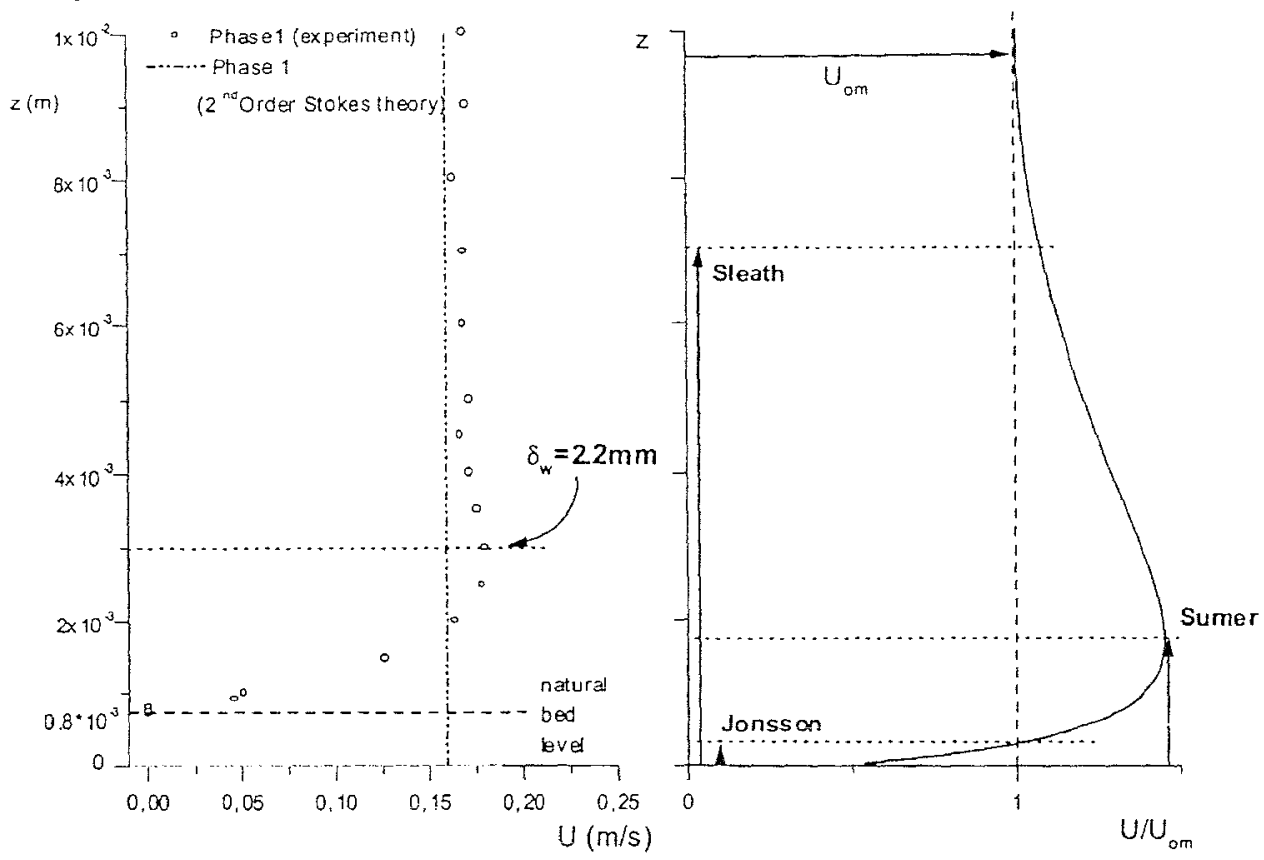

Fig.7 Profil expérimental et définitions de l'épaisseur de la couche limite Fig. 7 Definitions and profile of the boundary layer thickness

Le régime d'écoulement de la couche limite est caractérisée par le nombre de Reynolds ( $\mathrm{Re}=\frac{\mathbf{U}_{\mathrm{om}}{ }^{* a}}{v}$ ) et le rapport entre l'élongation horizontale, a, et la rugosité du fond, $\mathrm{k}$. La

détermination de la longueur de rugosité est une tâche difficile à partir de données expérimentales. Dans le cas d'une paroi rugueuse, $z_{0}$ est défini comme $U\left(z_{0}\right)=0 . R e_{k}$ représente $n$ "nombre de Reynolds rugueux": $\operatorname{Re}_{\mathrm{k}}=\frac{\mathrm{U}_{\mathrm{om}}{ }^{*} \mathrm{k}}{\mathrm{v}}$. Ici, $R_{\mathrm{ek}}=4.1 .10^{4}$, ce qui correspond à un type d'écoulement turbulent rugueux d'après les classifications de Kamphuis (1975). Pour notre cas d'étude (vague $W_{1}$ ), une épaisseur de couche limite $\delta_{W}$ peut être mesurée à partir des données expérimentales. $\delta_{W}$ s'étend sur $2.2 \mathrm{~mm}$ pour la phase de crête. En régime turbulent, Jonsson (1976) établit une équation théorique de cette épaisseur pour une paroi semi-rugueuse: $\delta_{\mathrm{j}}=0.072 \cdot\left(\mathrm{a}^{3} \mathrm{k}\right)^{1 / 4}$, où $\mathrm{k}$ représente un paramètre de rugosité de la paroi (dans notre cas, $\mathrm{k}=30 . \mathrm{z}_{0}=24 \mathrm{~mm}$ ). On en déduit $\delta_{\mathrm{J}}=3.4 \mathrm{~mm}$, ce qui semble adéquat avec les résultats expérimentaux. Fredsoe (1984) prend en compte l'écoulement au repos lors de chaque demi-période et assume un profil logarithmique des vitesses dans la couche limite. Trowbridge (1984) étendit son modèle en considérant une évolution temporelle de l'épaisseur de la couche limite. Le but de nos analyses futures est d'exprimer, à partir des résultats expérimentaux, l'épaisseur de la couche limite sous une forme dépendante du temps. En effet, le profil de vitesse est de forme logarithmique seulement pour les phases extrêmes et présente un "effet de mémoire" lors des phases d'accélération ou de décélération des vagues. la figure suivante montre les résultats expérimentaux obtenus par Vélocimétrie Doppler Laser: 

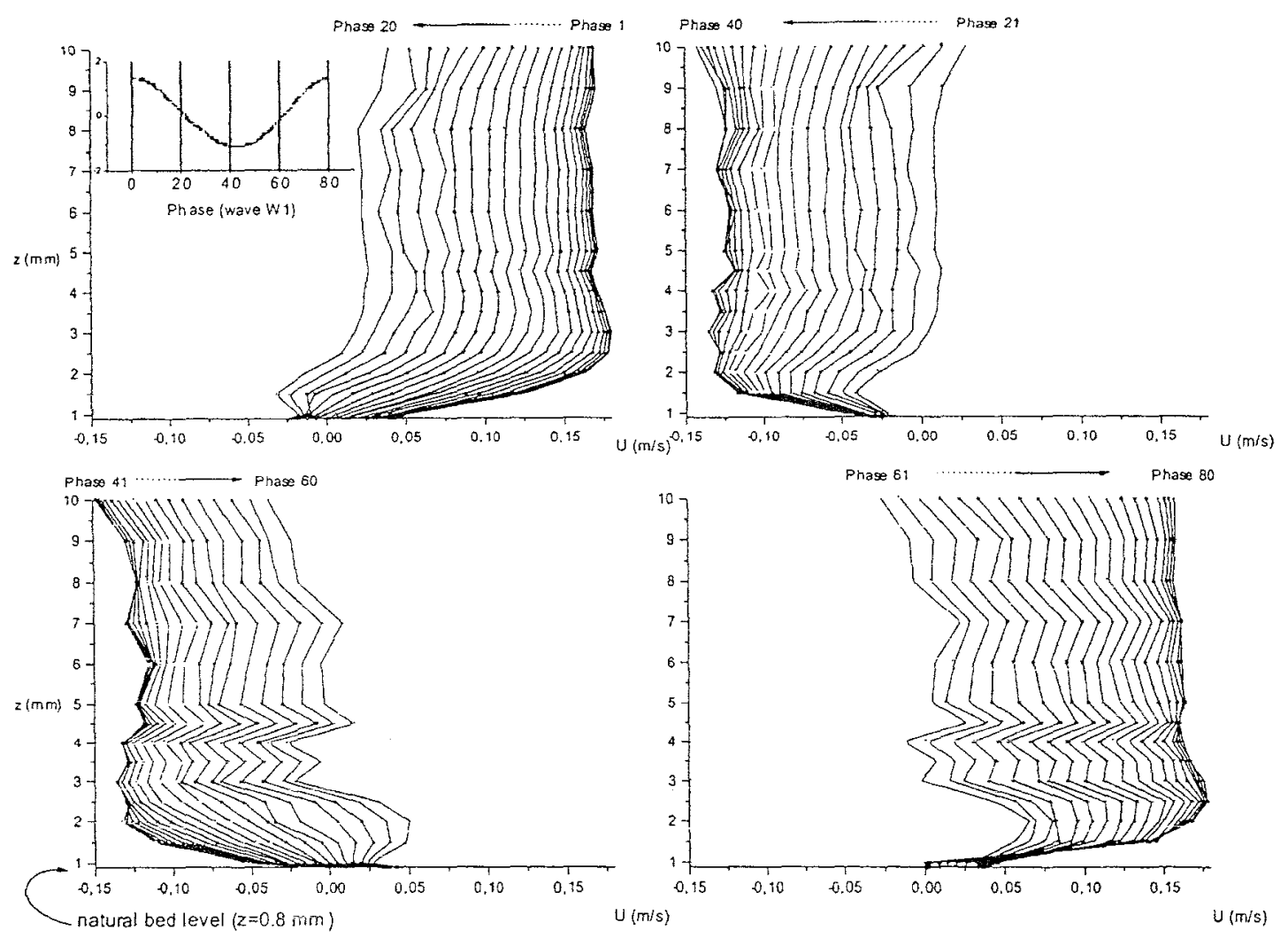

Fig. 8 Evolution des couches limites de vague en fonction de la phase (vague $W_{1}$ )

Fig. 8 Evolution of wave boundary layers on the bed with phase (wave $W_{i}$ )

Le graphe précédent (Fig.8) montre la diminution de l'épaisseur de la couche limite pour les phases entre crête et creux de la vague. Pour une ordonnée comprise entre 2 et $4 \mathrm{~mm}$, la présence de turbulence engendrerait une surélévation des vitesses. La remarque la plus intéressante concerne le double point d'inflexion des profils de vitesse pour les phases $20,25,60,65$ et 70 . Cette caractéristique révèle la présence d'une séparation. Le fluide "décolle" du fond du canal et crée une bulle de recirculation. Les pics de vitesse, observés très proche de la paroi, pourraient révéler une sous-couche très fine sur cette surface. Cependant, une explication plus plausible serait la création d'un artefact provoqué par la réflexion intense des faisceaux lumineux par le fond du canal. Par conséquent, on définit l'origine de l'axe des ordonnées à $\mathrm{z}_{0}=0.8 \mathrm{~mm}$.

\subsection{Modèle d'oscillateur amorti par frottement visqueux}

Beaucoup d'études de la couche limite s'effectuent sur une plaque plane dans un écoulement continu ou oscillant. Le profil des vitesses est alors bien connu et correspond à une position d'équilibre stable pour les positions extrêmes du mouvement. Les spécificités de la houle (accélération / décélération, composante verticale de la vitesse, ...) perturbent cet état d'équilibre. Ces perturbations vont engendrer l'oscillation de certains profils de vitesse pendant les phases de gradients élevés. Puis de nouvelles positions d'équilibre sont atteintes lors de la crête et du creux de la vague. On peut imaginer que le comportement d'une masse de fluide prés du fond est analogique à celui d'un oscillateur amorti dans toutes directions par frottement visqueux. Si l'on admet que la force de frottement est proportionnelle à la vitesse, l'évolution de cette dernière peut être écrite sous cette forme: 


$$
\mathbf{U}(\boldsymbol{z}, \phi)=\mathbf{P}_{1} \cdot \mathrm{e}^{\left(-\boldsymbol{z} / \mathbf{P}_{2}\right)} \cdot \cos \left(\mathbf{P}_{3} \cdot \boldsymbol{z}+\mathbf{P}_{4}\right)+\mathbf{U}_{\text {om }}, \quad \text { avec } \phi \text { la phase de la houle }
$$

Le paramètre $P_{1}$ dépend des conditions limites du profil de vitesse; $P_{2}$ dépend du temps de relaxation du mouvement; $P_{3}$ est la pseudo-période et $P_{4}$ la phase propre du profil.

Les paramètres $P_{i}$ doivent être déterminés en adéquation avec les autres tests afin de chercher une relation (temporelle, spatiale) avec les caractéristiques des vagues. Ceci peut permettre d'améliorer le modèle qui reste encore fébrile vu l'état d'avancement de l'analyse des données expérimentales. Il parait alors possible d'écrire l'évolution de la couche limite sous une forme dépendante du temps (ou phase) en interconnection avec les spécificités des vagues. Les figures suivantes (Fig.9 \&

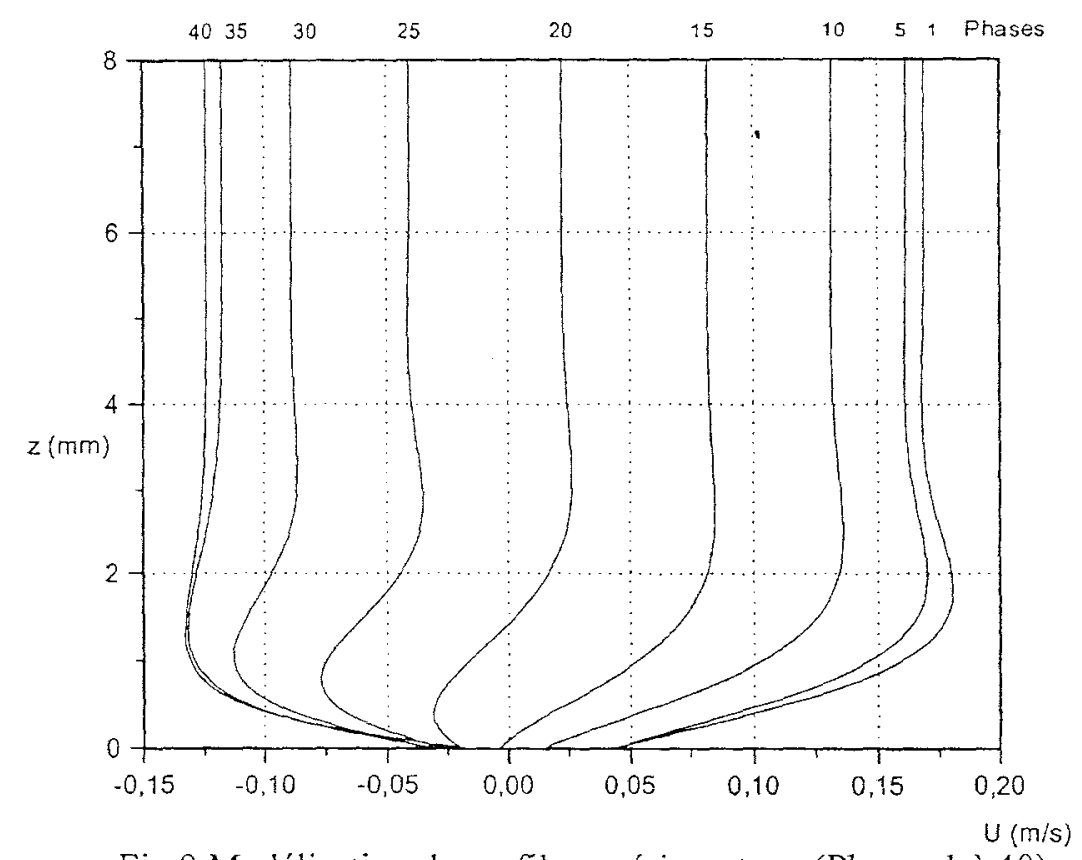

Fig.9 Modélisation de profils expérimentaux (Phases 1 à 40)

Fig.9 Oscillating wave modeling of the experimental velocity 10) présentent l'application du modèle à des données expérimentales (vague $W_{1}$, Fig.8) en utilisant une régression non-linéaire pour chaque phase de l'écoulement.

La modélisation permet une meilleure compréhension de la formation de la couche limite de la vague $W_{1}$. La quantification de la couche limite, en temps (phase de la vague) et en espace, paraitt possible mais présente toutefois des difficultés par le choix d'une définition donnée dans la littérature (Fig.7). Ainsi, d'après la définition de Sleath, l'épaisseur de couche limite varie entre 4 et $8 \mathrm{~mm}$, ou bien entre 0.5 et $2.5 \mathrm{~mm}$ selon Sumer, et entre 0 et $2.5 \mathrm{~mm}$ selon Jonsson. Malgré tout, la

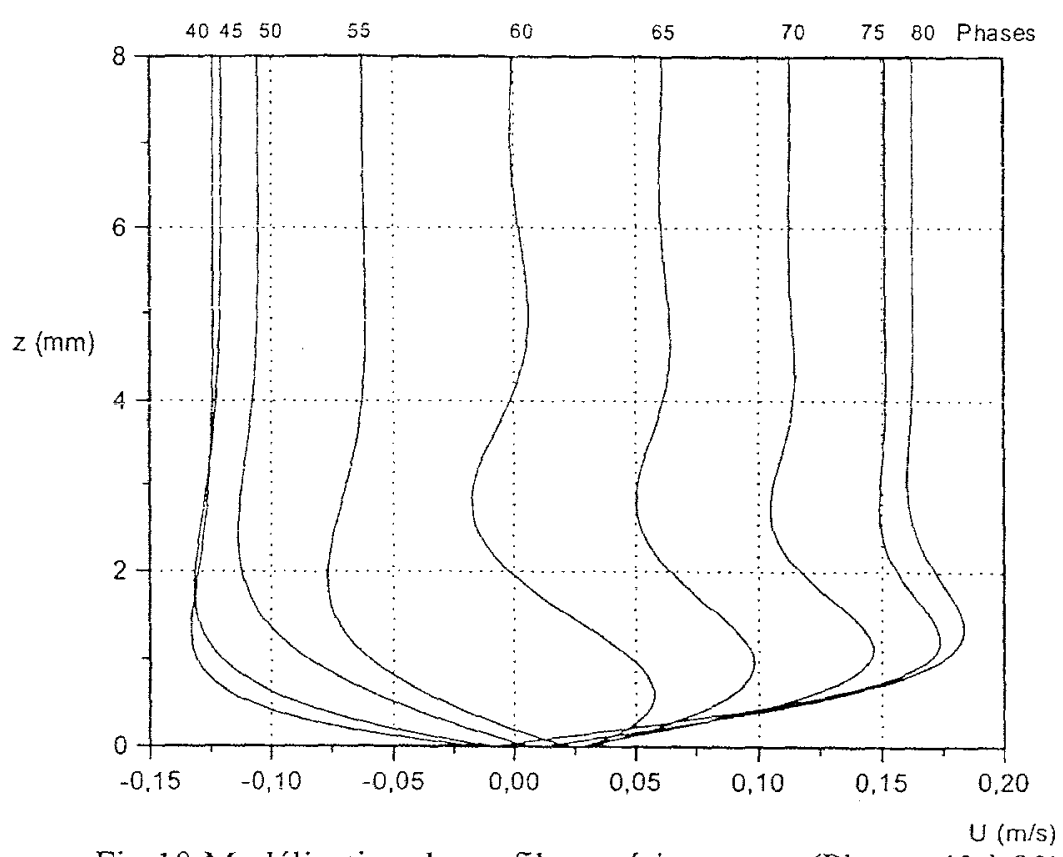

Fig.10 Modélisation de profils expérimentaux (Phases 40 à 80 ) Fig.10 Oscillating wave modeling of the experimental velocity périodicité de la couche limite suit la demi-période de la vague. 


\section{Analyse de la couche limite autour du cylindre}

L'étude se focalise sur le cas d'un écoulement de séparation sur le cylindre $(\mathrm{KC}=$ 1.5). Pour cette condition, on note la présence de vortex de Honji (1981). Le profil des vitesses prés de la surface du cylindre est asymétrique relativement à deux demipériodes consécutives. Le module de la vitesse est plus élevé lorsque la surface libre converge vers la crête que dans la direction opposée. Il en résulte l'émergence d'une cellule (ou zone) de recirculation sur la surface du cylindre ("steady streaming"). Le graphe suivant (Fig.11) montre les profils temporels et globaux des vitesses pour le rayon à la verticale supérieure du cylindre $\left(\theta=0^{\circ}\right.$, cf. Fig.2). On peut remarquer une dispersion des vitesses prés de la surface du cylindre $(\mathrm{z} \leq 3 \mathrm{~mm})$ particulièrement dans la phase d'accélération finale pour aitteindre la crête de la vague. Ce phénomène peut être du à une formation ou une croissance intermittente de la turbulence. Le taux de données n'est malheureusement pas suffisant pour espérer une étude précise des taux de turbulence.

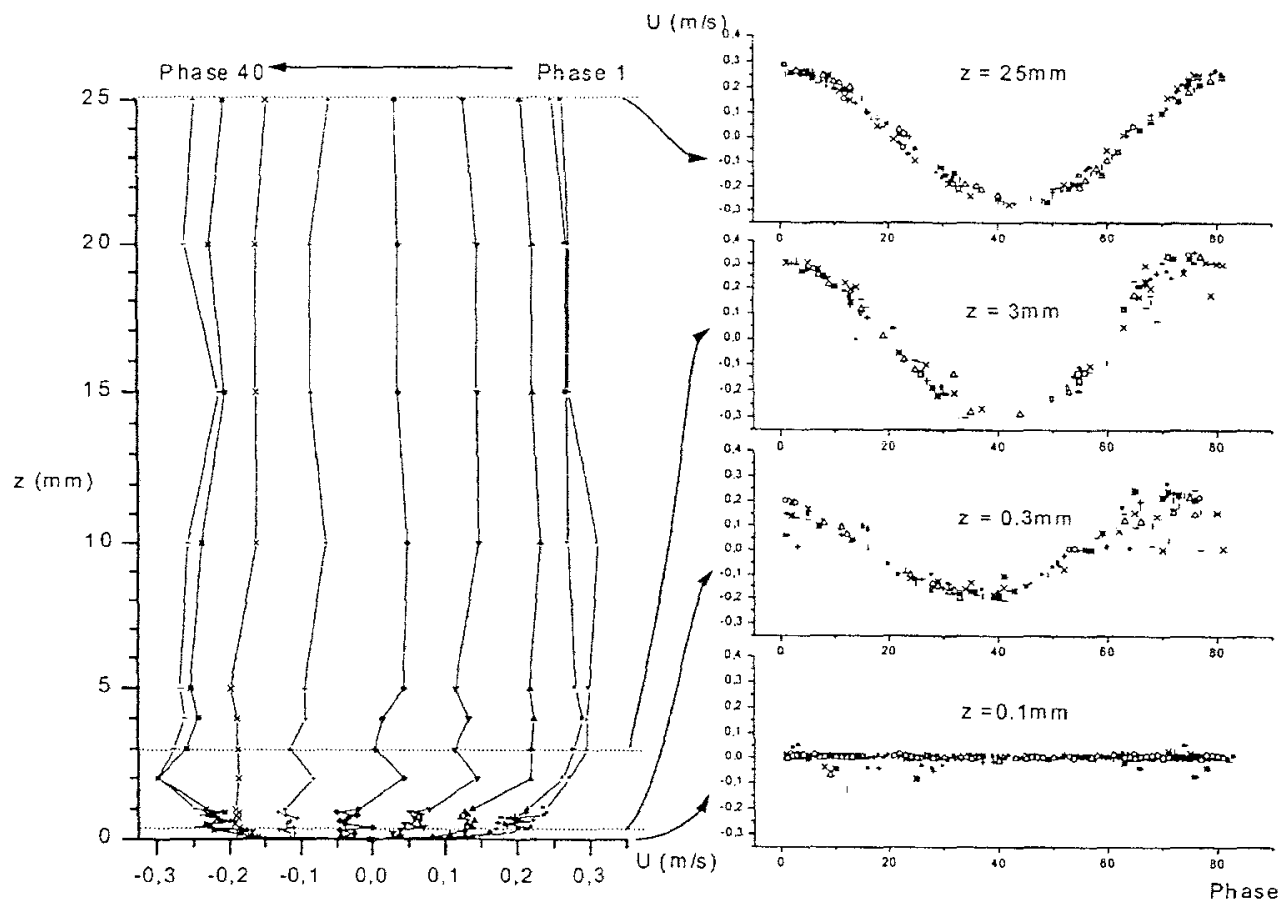

Fig. 11 Profils temporels et globaux des mesures de vitesse $\left(\theta=0^{\circ}, \mathrm{e} / \mathrm{D}=0.5\right)$

Fig. 11 Global and temporal velocity profiles for $\left(\theta=0^{\circ}\right.$, e/D=0.5)

On peut comparer les résultats expérimentaux (Fig.12) et l'application du modèle de l'oscillateur cité précédemment (Fig.13). Cette modélisation permet aussi une meilleure compréhension de la croissance de la couche limite au dessus de la structure. Son épaisseur est estimée à $3 \mathrm{~mm}$ pour la phase de crête conformément à la définition de Sumer. Schlichting (1979) définit l'épaisseur de couche limite comme une fonction du nombre de Reynolds $\operatorname{Re}_{\mathrm{D}}: \frac{\delta}{\mathrm{D}}=\mathbf{f}\left(\frac{1}{\sqrt{\operatorname{Re}_{\mathrm{D}}}}\right)$, avec $\operatorname{Re}_{\mathrm{D}}=\frac{\mathrm{U}_{\mathrm{om}}{ }^{*} \mathrm{D}}{v}$,

Les résultats expérimentaux donnent $\frac{\delta}{\mathrm{D}}=\frac{2.7}{\sqrt{\mathrm{Re}_{\mathrm{D}}}}$, ce qui est en bon agrément avec

l'étude de Jensen (1986) dans un tunnel oscillant. 

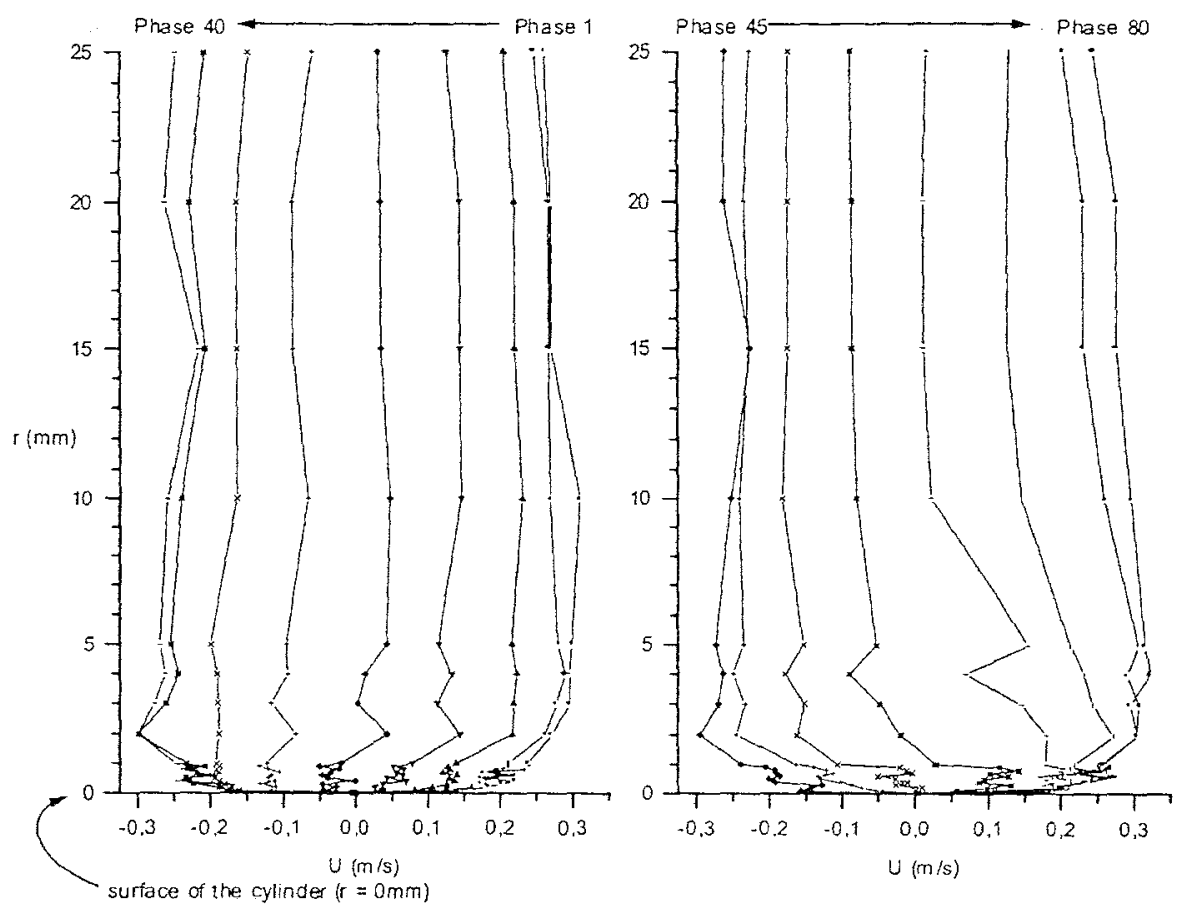

Fig. 12 Couche limite sur un rayon du cylindre $\left(\theta=0^{\circ}, \mathrm{e} / \mathrm{D}=0.5\right)$

Fig.12 Boundary layer profiles over the surface of the cylinder $\left(\theta=0^{\circ}, e / D=0.5\right)$ (experimental data)

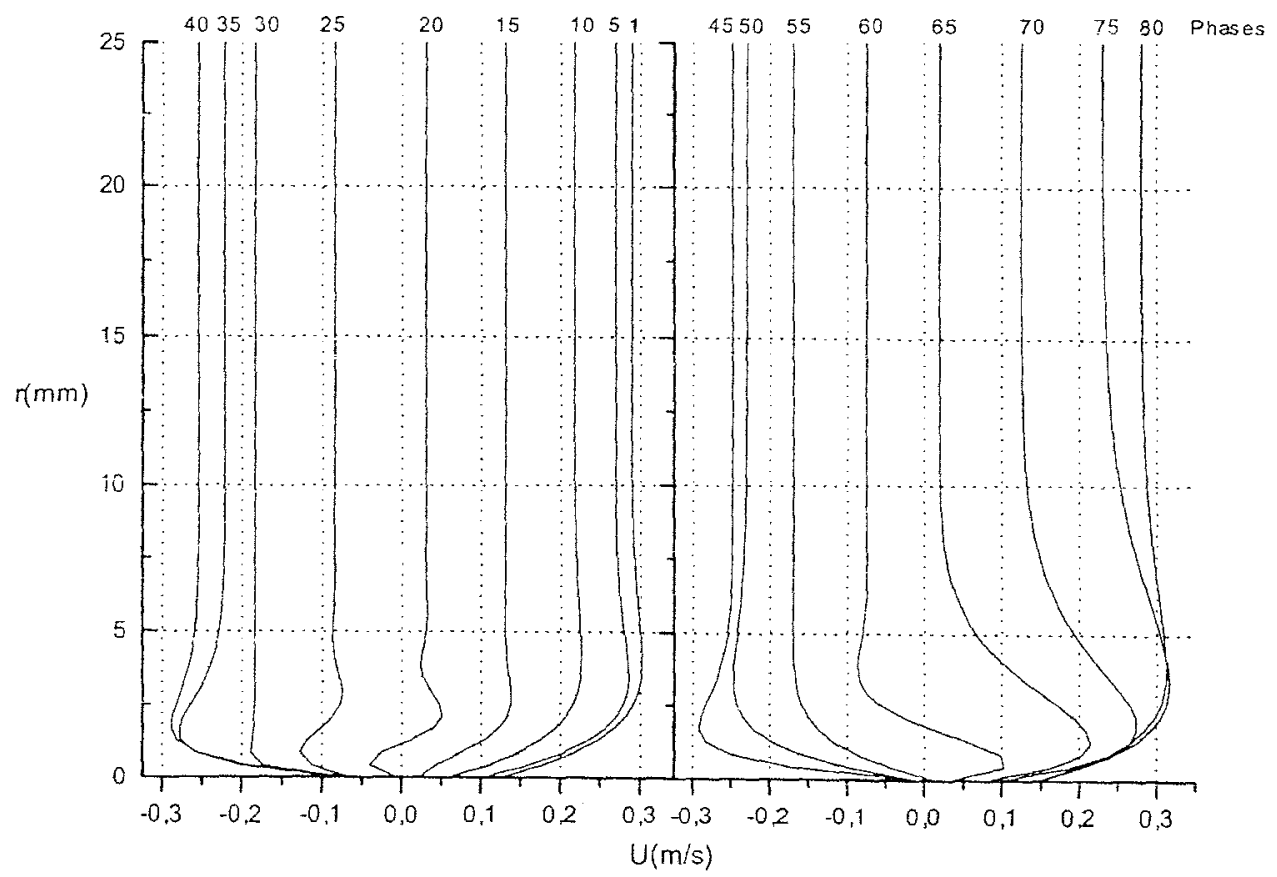

Fig.13 Modèle de couche limite sur la surface d'un cylindre $\left(\theta=0^{\circ}\right.$, e/D $\left.=0.5\right)$

Fig. 13 Boundary layer model over the surface of the cylinder $\left(\theta=0^{\circ}, e / D=0.5\right)$

L'analyse des profils révèle l'occurrence d'une cellule de séparation de fine épaisseur pour les phases 20 et 60 (Fig.14). En étudiant le développement des vitesses pour les autres rayons, l'épaisseur de cette zone est évaluée à $1 \mathrm{~mm}$. Cette valeur est très faible comparativement au diamètre du cylindre $(210 \mathrm{~mm})$ et on peut imaginer sa 
difficulté de détection pour de plus petits diamètres. Du comportement de cette recirculation (extension, déplacement, oscillation) dépendra le détachement tourbillonnaire pour des nombres de Keulegan-Carpenter plus élevés. L'analyse révèle que cette cellule se forme entre les phases 15 et 25 , puis disparait, pour se reformer à la phase 60 . Dans ce dernier cas, l'épaisseur de la recirculation est de $2 \mathrm{~mm}$.

\section{Conclusion}

La première partie des résultats donne des informations à propos des caractéristiques de la couche limite d'une vague. L'introduction d'un modèle d'oscillateur amorti par frottement visqueux est intéressante mais demande a être approfondie par l'analyse de l'ensemble des données expérimentales. L'étude des autres
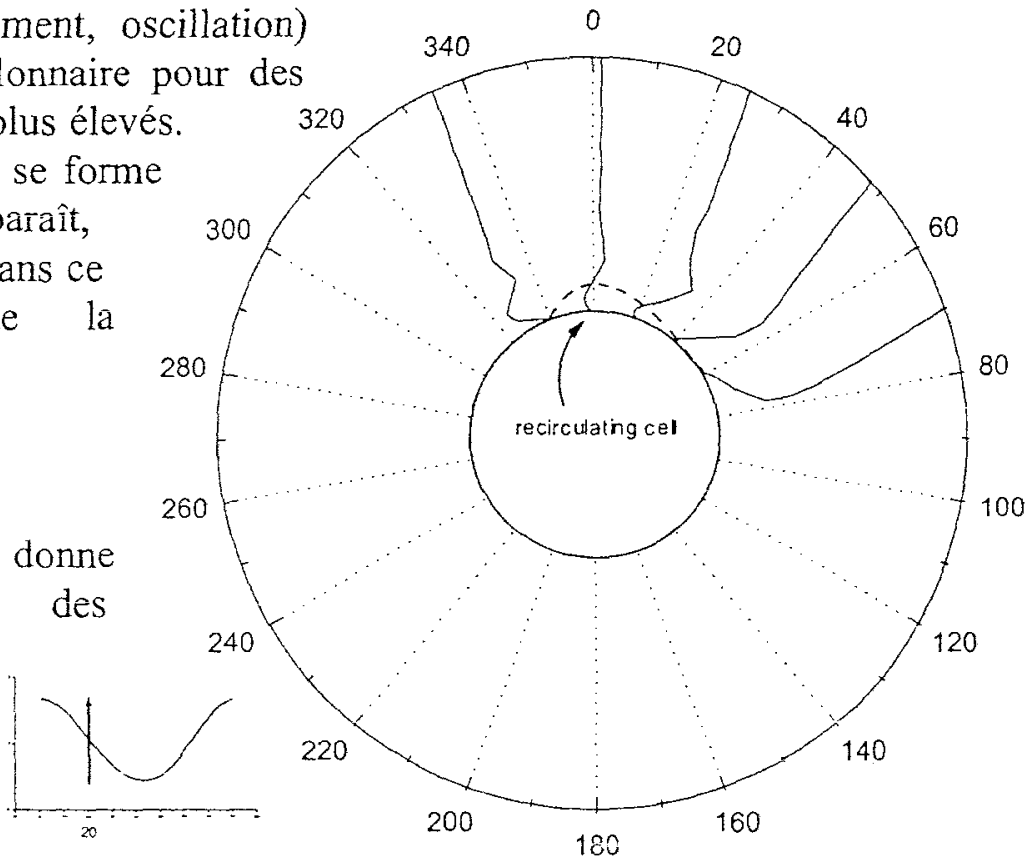
tests devrait donner plus d'informations concernant la formation de la couche limite autour du cylindre en fonction des paramètres des vagues et de l'influence du fond.

\section{Références}

CHAPLIN J.R. (1981) "Boundary layer separation from a cylinder in waves" International Symposium on Hydrodynamics in Ocean Engineering, Norwegian Institute of Technology, Band 1, pp. 645-666

DURSTHOFF W., MOUAZE D. (1997) "Development of a LASER Doppler system for measuring the velocity field around a cylinder placed close to the bottom" ISOPE-97

FREDSøE J. (1984) "Turbulent boundary layer in wave-current motion" Journal of Hydraulic Engineering, Vol. 110, Paper $N^{\circ} 19055$, pp. 1103-1120

FREDSØE J., JUSTESEN P. (1986) "Turbulent separation around cylinder in waves" Journal of Waterway, Port, Coastal and Ocean Engineering, Vol. 112, N², Paper N²0464, pp. 217-233

GIBSON M.M, JONES W.P., YOUNIS B.A. (1981) "Calculation of turbulent boundary layers on curved surfaces" The Physics of Fluids, Vol. 24, N³, pp. 386-395

JENSEN B.L., SUMER B.M. (1986) "Boundary layer over a cylinder placed near a wall" Institut of Hydrodynamic and Hydraulic Engineering., Progress Report, Vol. 64, pp. 31-39

JONSSON I.G. (1980) "A new approach to oscillatory rough turbulent boundary layers" Ocean Engineering, Vol. ${ }^{\circ}$, pp. $109-152$

JONSSON I.G., CARLSEN N.A. (1976) "Experimental and theoretical investigations in an oscillatory turbulent layer" EUROMECH Colloquium 215

KAMPHUIS J.W. "Friction factor under oscillatory waves" Journal of the Waterway, Port and Ocean Division, Transactions of the ASCE, Vol. 101, NoWW2, pp. 135-144

SAKOUT A., JARNO-DRUAUX A., BELORGEY M. (1990) "Contribution à l'étude de l'érosion induite par la houle au voisinage d'un cylindre placé à proximité du fond" Génie Civil - Génie Côtier, pp. ${ }^{\circ} 40-57$

SCHLICHTING H. (1979) "Boundary-layer theory" $7^{\text {th }}$ ed., McGraw-Hill Book Co.

SLEATH J.F.A. (1987) "Turbulent oscillatory flow over rough beds" Journal of Fluid Mechanics, Vol. ${ }^{\circ} 182$, pp. $369-409$ 